BMJ Open

Diabetes

Research

\& Care

\title{
Clinical characteristics and outcomes of patients with end-stage renal disease hospitalized with diabetes ketoacidosis
}

Rodolfo J Galindo (D , ${ }^{1}$ Francisco J Pasquel, ${ }^{1}$ Maya Fayfman, ${ }^{1}$ Katerina Tsegka, ${ }^{1}$ Neil Dhruv, ${ }^{1}$ Saumeth Cardona, ${ }^{1}$ Heqiong Wang, ${ }^{2}$ Priyathama Vellanki (D) , Guillermo E Umpierrez (1) ${ }^{1}$

To cite: Galindo RJ, Pasquel FJ, Fayfman M, et al. Clinical characteristics and outcomes of patients with end-stage renal disease hospitalized with diabetes ketoacidosis. BMJ Open Diab Res Care 2020;8:e000763. doi:10.1136/ bmjdrc-2019-000763

- Additional material is published online only. To view please visit the journal online (http://dx.doi.org/10.1136/ bmjdrc-2019-000763).

Part of this study was presented at the 78th Scientific Sessions of the American Diabetes Association, Orlando, June 2018.

Received 17 July 2019 Revised 17 December 2019 Accepted 17 January 2020

Check for updates

\section{(c) Author(s) (or their} employer(s)) 2020. Re-use permitted under CC BY. Published by BMJ.

${ }^{1}$ Division of Endocrinology, Emory University School of Medicine, Atlanta, Georgia, USA ${ }^{2}$ Rollins School of Public Health, Emory University, Atlanta, Georgia, USA

\section{Correspondence to} Professor Guillermo E Umpierrez; geumpie@emory.edu

\section{ABSTRACT}

Introduction There is limited evidence to guide management in patients with end-stage renal disease (ESRD) on chronic hemodialysis admitted with diabetes ketoacidosis. Thus, we investigated the clinical characteristics and outcomes of patients with ESRD admitted with diabetic ketoacidosis (DKA).

Methods In this observational study, we used International Classification of Diseases Ninth/Tenth Revision codes to identify adult (aged 18-80 years) patients admitted to Emory University Hospitals between 1 January 2006 and 31 December 2016. DKA and ESRD diagnoses were confirmed by reviewing medical records and by admission laboratory results.

Results Among 307 patients with DKA meeting the inclusion and exclusion criteria, 22.1\% (n: 68) had ESRD on hemodialysis and $77.9 \%$ ( $\mathrm{n}$ : 239) had preserved renal function (estimated glomerular filtration rate $>60 \mathrm{~mL}$ / $\left.\min / 1.73 \mathrm{~m}^{2}\right)$. Compared with patients with preserved renal function, the admission blood glucose was higher $(804.5 \pm 362.6 \mathrm{mg} / \mathrm{dL}$ vs $472.5 \pm 137.7 \mathrm{mg} / \mathrm{dL})$ and the mean hemoglobin A1c was lower $(9.6 \% \pm 2.1$ vs $12.0 \% \pm 2.5)$ in patients with DKA and ESRD, both $p<0.001$. The rates of hypoglycemia $<70 \mathrm{mg} / \mathrm{dL}(34 \%$ vs $14 \%, \mathrm{p}=0.002)$ and $<54 \mathrm{mg} / \mathrm{dL}(13 \%$ vs $5 \%, \mathrm{p}=0.04)$ were higher in the ESRD group. During hospitalization, more patients with ESRD develop volume overload ( $28 \%$ vs $3 \%, p<0.001)$ and require mechanical ventilation ( $24 \%$ vs $3 \%, p=<0.001)$. There were no differences in hospital mortality ( $3 \%$ vs $0 \%, p=0.21$ ), but length of stay (median 7.0 vs 3.0 days, $p<0.001$ ) was longer in the ESRD cohort. After adjusting for multiple covariates, patients with DKA and ESRD have higher odds of hypoglycemia (OR $3.3,95 \% \mathrm{Cl} 1.51$ to 7.21 , $\mathrm{p}=0.003$ ) and volume overload (OR 4.22, 95\% Cl 1.37 to $13.05, p=0.01$ ) compared with patients with DKA with preserved renal function.

Conclusions Patients with DKA and ESRD on chronic hemodialysis had worse clinical outcomes including higher rates of hypoglycemia, volume overload, need for mechanical ventilation and longer length of stay, compared with patients with preserved kidney function.

\section{INTRODUCTION}

In 2015, there were almost 700000 patients with end-stage renal disease (ESRD) in the USA. ${ }^{12}$ Diabetes is a leading cause of chronic kidney disease and ESRD, with approximately

\section{Significance of this study}

What is already known about this subject?

- There is limited evidence on the management and outcomes of patients with end-stage renal disease (ESRD) admitted with diabetic ketoacidosis (DKA).

What are the new findings?

- Compared with patients admitted with DKA and preserved renal function, patients with ESRD (1) presented with higher admission blood glucose, but lower hemoglobin A1c; (2) had higher rates of hypoglycemia, volume overload and need for mechanical ventilation; and (3) have similar mortality, but longer length of stay and higher hospital costs.

How might these results change the focus of research or clinical practice?

- The poor clinical outcome observed in patients with ESRD highlights the need for close glucose monitoring and a personalized approach to volume replacement during treatment for DKA; as well as the need for prospective studies to assess personalized management algorithms to improve treatment-related complications and reduce healthcare utilization in this high-risk population.

$40 \%-50 \%$ of patients on dialysis having a diagnosis of diabetes. ${ }^{1-3}$ The adjusted survival after 3 and 5 years of hemodialysis initiation is $55 \%$ and $40 \%$, with patients with diabetes having the worst adjusted survival rates. ${ }^{2-4}$

Diabetic ketoacidosis (DKA $)^{5}$ is a serious and life-threatening hyperglycemic emergency that occurs in patients with type 1 and type 2 diabetes. ${ }^{6}$ In a nationwide study in the USA, we recently reported that up to $7.5 \%$ of patients admitted with DKA had ESRD listed as a comorbidity. ${ }^{6}$ The diagnosis and management of DKA in patients with ESRD is challenging due to alterations in glucose metabolism, insulin sensitivity and altered renal clearance of antidiabetic medications. ${ }^{38}$ Despite extensive data from landmark studies on the management of DKA 
in general populations, ${ }^{69}$ there is virtually no evidence to guide appropriate volume replacement, insulin and potassium therapy in patients with DKA and ESRD. ${ }^{10} \mathrm{We}$ recently reported that ESRD in patients with DKA was associated with higher adjusted risk for all-cause readmissions, with most readmissions occurring within 2 weeks after discharge. ${ }^{6}$

A major limitation of prior studies has been the use of International Classification of Diseases Ninth/Tenth Revision (ICD-9/10) codes to identify the diagnosis of DKA, without confirmation of the diagnosis based on admission biochemical/laboratory data. ${ }^{6} 9$ Accordingly, we performed a detailed analysis of clinical and laboratory characteristics to confirm the presence of DKA and clinical outcomes in patients with ESRD on hemodialysis admitted to our tertiary referral academic center. We hypothesized that patients with ESRD, presenting with DKA, will have increased morbidity and treatmentrelated complications compared with patients with DKA and preserved kidney function.

\section{METHODS}

\section{Study population}

We included adult patients between 18 and 80 years of age, with admission ICD-9/10 codes for DKA and ESRD on chronic maintenance hemodialysis, hospitalized at Emory University Hospitals between 1 January 2006 and 31 December 2016. We excluded pregnant patients, those not meeting the laboratory diagnosis. ${ }^{11}$

\section{Cohort creation}

This retrospective study searched admission data via the Emory Clinical Data Warehouse. After identifying patients with admission diagnosis of DKA and ESRD on hemodialysis by prespecified ICD-9/10 criteria (see online supplementary materials), we included only the first admission. We then confirmed the diagnosis of DKA for all subjects by using prespecified laboratory diagnostic criteria set by the American Diabetes Association (ADA; see online supplementary materials). ${ }^{11}$ The diagnosis of ESRD was confirmed by laboratory data (estimated glomerular filtration rate $\left(\right.$ eGFR) $<15 \mathrm{~mL} / \mathrm{min} / 1.73 \mathrm{~m}^{2}$ ) on admission. For comparison, we included patients with DKA and preserved renal function with eGFR $>60 \mathrm{~mL} /$ $\min / 1.73 \mathrm{~m}^{2}$.

\section{Study variables}

We collected patient's demographics: age, gender, race, ethnicity, body mass index, admission, and inpatient laboratory values including hemoglobin Alc (HbAlc), glucose, $\mathrm{pH}$, ketones, total serum osmolality $(2(\mathrm{Na})+18$ / glucose+blood urea nitrogen/2), anion gap, serum sodium, serum potassium, and eGFR.

Comorbidities and hospital complications were identified by ICD-9/10 codes generated during the hospitalization as previously described. ${ }^{12}$ ICD-9 codes generated during the hospital stay, but not present during admission, and laboratory data generated during the stay were used to calculate the rate of hospital-related complications, including: hypokalemia $(<3 \mathrm{mmol} / \mathrm{L})$, severe hypokalemia $(<2.5 \mathrm{mmol} / \mathrm{L})$, myocardial infarction, volume overload (pulmonary edema or congestive heart failure), need for mechanical ventilation, deep vein thrombosis, pulmonary thromboembolism, sepsis, and rhabdomyolysis. We report all-cause mortality during hospitalization, and following 30 and 365 days after discharge. We also analyzed length of hospital stay (LOS) and hospitalization costs. Response to treatment was analyzed as time to normalization of glucose (blood glucose (BG) $<250 \mathrm{mg} / \mathrm{dL}$ ) during treatment, rates of hypoglycemia $<70 \mathrm{mg} / \mathrm{dL},<54 \mathrm{mg} / \mathrm{dL}$ and $<40 \mathrm{mg} / \mathrm{dL}$, rates of hypokalemia, mean daily glucose, etc. BG values included point-of-care testing and laboratory glucose measures. Patients were managed by their primary care teams with a nurse-driven protocol available across all Emory Hospitals, based on the ADA guidelines for DKA management.

\section{Statistical analysis}

Major outcomes of interest included frequency of hypoglycemia, volume overload, hypokalemia, LOS, and mortality during hospitalization between patients with DKA and ESRD and with preserved kidney function. Descriptive statistics were used to compare clinical and biochemical characteristics on admission and during hospitalization. The comparisons were made with the use of Wilcoxon tests for continuous variables and $\chi^{2}$ tests (or Fisher's exact tests) for discrete variables. Multivariate logistic regression analysis was conducted for outcome hypoglycemia and volume overload, which was adjusted by age, sex, race, body mass index (and relevant comorbidities). A $p$ value $<0.05$ was considered significant. All analyses were performed using SAS software V.9.4 (SAS Institute). The data are generally presented as mean $\pm \mathrm{SD}$ for continuous variables and $\mathrm{n}(\%)$ for discrete variables.

\section{RESULTS}

Among 307 patients with DKA meeting the inclusion and exclusion criteria, a total of $68(22.1 \%)$ patients had ESRD on chronic hemodialysis and 239 (77.9\%) patients had preserved renal function (eGFR $>60 \mathrm{~mL}$ / min). Baseline demographic characteristics and admission laboratory results are shown in table 1 . There were no racial differences between groups, with the majority being African-American for both ( $71 \%$ vs $70 \%, p=0.88)$, but patients with ESRD and DKA were older (mean $( \pm$ SD) age: $54.9 \pm 16.5$ vs $35.6 \pm 14.1$ years, $\mathrm{p}<0.001)$, more likely to be female $(66 \%$ vs $51 \%, \mathrm{p}=0.02)$, and to have government-sponsored (Medicare/Medicaid) insurance ( $74 \%$ vs $29 \%, \mathrm{p}<0.01)$ compared with patients with DKA and preserved renal function.

Despite having a lower mean HbA1c on admission $(9.6 \% \pm 2.1$ vs $12 \% \pm 2.5, \mathrm{p}<0.001)$, the ESRD cohort had higher admission mean BG $(804.5 \pm 362.6 \mathrm{mg} / \mathrm{dL}$ vs $472.5 \pm 137.7 \mathrm{mg} / \mathrm{dL}, \mathrm{p}<0.001)$, as well as higher mean BG during the first 48 hours of treatment compared 


\begin{tabular}{|c|c|c|c|}
\hline Characteristic & $\begin{array}{l}\text { ESRD } \\
\mathrm{n}=68 \\
(22.1 \%)\end{array}$ & $\begin{array}{l}\text { No ESRD } \\
\mathrm{n}=239 \\
(77.9 \%)\end{array}$ & $P$ value \\
\hline Age (years) & $54.9 \pm 16.5$ & $35.6 \pm 14.1$ & $<0.001$ \\
\hline Sex, (n) \% & & & 0.02 \\
\hline Female & $45(66)$ & $121(51)$ & \\
\hline Male & $23(34)$ & $118(49)$ & \\
\hline Body mass index $\left(\mathrm{kg} / \mathrm{m}^{2}\right)$ & $27.8 \pm 8.3$ & $27.1 \pm 7.3$ & 0.43 \\
\hline Race, (n) \% & & & 0.88 \\
\hline Caucasian & $18(26)$ & $59(25)$ & \\
\hline African-American & $48(71)$ & $168(70)$ & \\
\hline Others & $2(3)$ & $12(5)$ & \\
\hline Ethnicity, (n) \% & & & 0.61 \\
\hline Hispanic & $1(1)$ & $9(4)$ & \\
\hline Non-Hispanic & $60(88)$ & $198(83)$ & \\
\hline Not reported & $7(10)$ & $32(13)$ & \\
\hline Insurance, (n) \% & & & $<0.001$ \\
\hline Commercial & $15(22)$ & $88(37)$ & \\
\hline $\begin{array}{l}\text { Government (Medicare/ } \\
\text { Medicaid) }\end{array}$ & $50(74)$ & $68(29)$ & \\
\hline No insurance & $3(4)$ & $82(34)$ & \\
\hline \multicolumn{4}{|l|}{ Comorbidities, n (\%) } \\
\hline Coronary artery disease & $14(21)$ & 7 (3) & $<0.001$ \\
\hline Hyperlipidemia & $22(32)$ & $34(14)$ & $<0.001$ \\
\hline $\begin{array}{l}\text { Chronic obstructive } \\
\text { pulmonary disease }\end{array}$ & $8(12)$ & $12(5)$ & 0.06 \\
\hline $\begin{array}{l}\text { Peripheral vascular } \\
\text { disease }\end{array}$ & $6(9)$ & $1(0.4)$ & $<0.001$ \\
\hline Acute pancreatitis & $2(3)$ & $10(4)$ & $>0.99$ \\
\hline Homelessness & 0 & $9(4)$ & 0.22 \\
\hline Depression & $10(15)$ & $27(11)$ & 0.53 \\
\hline Substance abuse & $2(3)$ & $18(8)$ & 0.27 \\
\hline
\end{tabular}

Data presented as mean $\pm(\mathrm{SD})$ or count (percentage), unless otherwise indicated.

ESRD, end-stage renal disease.

with the control group. In addition, patients with ESRD presented with higher anion gap $(23.6 \pm 7$ vs $20.1 \pm 4.5$, $\mathrm{p}=<0.001)$, serum osmolarity $(304.4 \pm 19.5$ vs $291.1 \pm 11.2$, $\mathrm{p}=<0.001)$, but lower beta-hydroxybutyrate $(4.5 \pm 3.3$ vs $6.2 \pm 2.3 \mathrm{mmol} / \mathrm{L}, \mathrm{p}=0.01$ ) levels compared with patients with DKA and preserved kidney function.

Metabolic parameters and response to medical therapy are shown in table 2 . The time to correction of hyperglycemia $(<250 \mathrm{mg} / \mathrm{dL})$ was significantly longer in the ESRD group (8.4 \pm 2.6 hours vs $7.2 \pm 3.1$ hours, $p=0.03$ ) compared with patients with preserved renal function. The rate of glucose reduction within the first 24 hours was higher in the ESRD group $(-596.1 \pm 315.2 \mathrm{mg} /$ $\mathrm{dL}$ vs $-268.0 \pm 154.3 \mathrm{mg} / \mathrm{dL}, \mathrm{p}=<0.001)$, and during the first 48 hours of therapy $(-617.3 \pm 374.9 \mathrm{mg} /$ $\mathrm{dL}$ vs $-256.1 \pm 160.6 \mathrm{mg} / \mathrm{dL}, \mathrm{p}=<0.001)$. The rates of hypoglycemia $<70 \mathrm{mg} / \mathrm{dL}(34 \%$ vs $14 \%, \mathrm{p}=<0.001)$ and $<54 \mathrm{mg} / \mathrm{dL}(13 \%$ vs $5 \%, \mathrm{p}=0.04)$ were twofold to threefold higher in the ESRD group compared with those with preserved renal function, but there were no differences in the rate of severe hypoglycemia $<40 \mathrm{mg} / \mathrm{dL}$ between groups (figure 1 ).

Patients with ESRD presented with higher potassium levels $(5.8 \pm 1.4 \mathrm{mmol} / \mathrm{L}$ vs $4.6 \pm 0.9 \mathrm{mmol} / \mathrm{L}, \mathrm{p}<0.001)$. During treatment, a similar number of patients experienced hypokalemia $(<3 \mathrm{mmol} / \mathrm{L})$, with no statistically significant differences ( $24 \%$ vs $30 \%, \mathrm{p}=0.30)$ between groups.

Patients with DKA and ESRD experienced significantly higher rates of hospital complications including volume overload (28\% vs 3\%, $\mathrm{p}<0.001)$, myocardial infarction ( $10 \%$ vs $0.4 \%, \mathrm{p}=<0.001)$, sepsis $(22 \%$ vs $3 \%, \mathrm{p}<0.001)$, deep venous thrombosis ( $7 \%$ vs $1 \%, \mathrm{p}=0.01$ ), and need for mechanical ventilation ( $24 \%$ vs $3 \%, \mathrm{p}<0.001)$ compared with patients with DKA and preserved renal function, all $\mathrm{p}<0.001$ (table 3 ). The all-cause hospital mortality was low in both groups ( $3 \%$ vs $0 \%, p=0.21$ ), but was significantly higher in the ESRD cohort at 30 days $(10 \%$ vs $1 \%$, $\mathrm{p}<0.001)$ and at 1 year $(18 \%$ vs $1 \%, \mathrm{p}<0.001)$ compared with patients with preserved renal function. In addition, the LOS was significantly longer (median 7.0 days (IQR $4.0-12.5$ ) vs median 3.0 days (IQR 2.0-4.0), $\mathrm{p}<0.001$ ) and hospitalization costs were significantly higher in the ESRD cohort (median $\$ 50844$ (IQR \$31 503-\$77 730) vs median \$14252 (IQR \$10 003-\$23 886), p<0.001).

After adjusting for multiple variables including patient's age, sex, race, and body mass index, we found that patients with DKA and ESRD have higher odds of hypoglycemia compared with patients with DKA and preserved renal function (OR 3.3, 95\% CI 1.51 to 7.21 , $\mathrm{p}=0.003)$. Similarly, in multivariate analysis adjusting for age, sex, race, body mass index, and history of coronary artery disease, we found that patients with ESRD have also an increased odds for volume overload (OR 4.22, $95 \%$ CI 1.37 to $13.05, \mathrm{p}=0.01$ ).

\section{DISCUSSION}

In this study, we analyzed the clinical characteristics and outcomes of patients hospitalized with DKA and ESRD on chronic maintenance hemodialysis. We report significant metabolic and clinical outcome differences in patients with DKA and ESRD compared with patients with preserved renal function. The ESRD group presented with twofold higher glucose levels and higher admission potassium concentration compared with those with preserved renal function. We also observed a twofold higher rate of hypoglycemia and a 10-fold higher rate of volume overload, compared with patients with preserved renal function. In addition, patients with ESRD had higher healthcare resource utilization, including longer LOS and hospitalization costs compared with patients with DKA and preserved renal function. 
Table 2 Metabolic parameters on admission and response to medical therapy

\begin{tabular}{|c|c|c|c|}
\hline Metabolic parameters & ESRD & No ESRD & $P$ value \\
\hline HbA1c (\%) & $9.6 \pm 2.1$ & $12 \pm 2.5$ & $<0.001$ \\
\hline Glucose (mg/dL) & $804.5 \pm 362.6$ & $472.5 \pm 137.7$ & $<0.001$ \\
\hline Sodium (mmol/L) & $129.8 \pm 7.5$ & $132.4 \pm 4.9$ & $<0.001$ \\
\hline Osmolality (mOsm/kg) & $304.4 \pm 19.5$ & $291.1 \pm 11.2$ & $<0.001$ \\
\hline Potassium (mmol/L) & $5.8 \pm 1.4$ & $4.6 \pm 0.9$ & $<0.001$ \\
\hline Anion gap (mEq/L) & $23.6 \pm 7$ & $20.1 \pm 4.5$ & $<0.001$ \\
\hline Bicarbonate (mEq/L) & $12.6 \pm 4.0$ & $13.0 \pm 3.9$ & 0.47 \\
\hline Beta-hydroxybutyrate (mmol/L) & $4.5 \pm 3.3$ & $6.2 \pm 2.3$ & 0.01 \\
\hline $\mathrm{pH}$ & $7.1 \pm 0.2$ & $7.2 \pm 0.2$ & 0.42 \\
\hline \multicolumn{4}{|l|}{ Response to therapy } \\
\hline Average BG days $1-10(\mathrm{mg} / \mathrm{dL})$ & $268.9 \pm 86.3$ & $255.5 \pm 70.3$ & 0.35 \\
\hline Average $B G$ day 1 (mg/dL) & $629.4 \pm 304.6$ & $364.2 \pm 114.7$ & $<0.001$ \\
\hline Average $B G$ day $2(\mathrm{mg} / \mathrm{dL})$ & $213.8 \pm 124.1$ & $201.4 \pm 67.0$ & 0.25 \\
\hline Average $B G$ day $3(\mathrm{mg} / \mathrm{dL})$ & $216.5 \pm 106.0$ & $210.4 \pm 86.2$ & 0.93 \\
\hline Average $B G$ day $4(\mathrm{mg} / \mathrm{dL})$ & $192.1 \pm 107.5$ & $209.6 \pm 87.6$ & 0.04 \\
\hline Average BG day $5(\mathrm{mg} / \mathrm{dL})$ & $191.9 \pm 112.4$ & $204.3 \pm 100.5$ & 0.24 \\
\hline Average BG in first 48 hours & $427.2 \pm 203.2$ & $288.0 \pm 78.7$ & $<0.001$ \\
\hline Time to correction of hyperglycemia (BG $<250 \mathrm{mg} / \mathrm{dL}$ ), hours & $8.4 \pm 2.6$ & $7.2 \pm 3.1$ & 0.03 \\
\hline Glucose reduction in first 24 hours (mg/dL) & $-596.1 \pm 315.2$ & $-268.0 \pm 154.3$ & $<0.001$ \\
\hline Glucose reduction in first 48 hours (mg/dL) & $-617.3 \pm 374.9$ & $-256.1 \pm 160.6$ & $<0.001$ \\
\hline Hypoglycemia <70 mg/dL, n (\%) & $23(34)$ & $34(14)$ & 0.002 \\
\hline Hypoglycemia <54 mg/dL, n (\%) & $9(13)$ & $13(5)$ & 0.04 \\
\hline Hypoglycemia <40 mg/dL, n (\%) & $2(3)$ & $4(2)$ & 0.62 \\
\hline Hypokalemia <3 mEq/L, n (\%) & $16(24)$ & $71(30)$ & 0.30 \\
\hline Hypokalemia <2.5 mEq/L & $3(4)$ & $9(4)$ & 0.73 \\
\hline
\end{tabular}

Data presented as mean $\pm(\mathrm{SD})$ or count (percentage), unless otherwise indicated.

$\mathrm{BG}$, blood glucose; ESRD, end-stage renal disease.

Diabetes is a leading cause of ESRD, with about half of patients requiring dialysis having a diagnosis of diabetes. ${ }^{1-3}$ There is limited high-quality evidence on the management and outcomes of DKA in patients with ESRD. ${ }^{10}$ The poor clinical outcome observed in these patients highlights the need for close metabolic monitoring and intensified treatment regimens to prevent the development of DKA, as well as the need for prospective studies to assess personalized management algorithmsincluding volume replacement and insulin therapy-to improve treatment-related complications and reduce healthcare costs in this high-risk population.

The mainstay of DKA management in patients with type 1 and type 2 diabetes includes the administration of intravenous fluids, insulin and electrolyte replacement therapy. ${ }^{11}$ For patients without cardiac or renal compromise, current guidelines recommend infusing isotonic saline at a rate of $15-20 \mathrm{~mL} / \mathrm{kg}$ or $1-1.5 \mathrm{~L}$ during the first hour, with subsequent rate of $250-500 \mathrm{~mL} /$ hour. $^{11}$ The higher rates of complications observed in our study
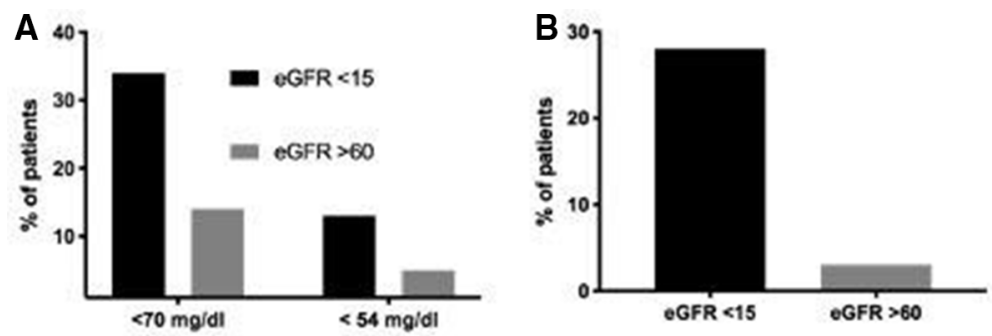

Figure 1 Proportion of patients developing $(A)$ hypoglycemia and $(B)$ volume overload after initiating therapy (all $p<0.05)$. (A) Hypoglycemia $<70$ and $<54 \mathrm{mg} / \mathrm{dL}$. (B) Volume overload. eGFR, estimated glomerular filtration rate. 
Table 3 Hospital complications and clinical outcomes

\begin{tabular}{lccc}
\hline Complications, $\mathbf{n}(\%)$ & ESRD & No ESRD & P value \\
\hline Volume overload & $19(28)$ & $7(3)$ & $<0.001$ \\
Mechanical ventilation & $16(24)$ & $6(3)$ & $<0.001$ \\
Sepsis & $15(22)$ & $8(3)$ & $<0.001$ \\
Myocardial infarction & $7(10)$ & $1(0.4)$ & $<0.001$ \\
Rhabdomyolysis & $3(4)$ & $0(0)$ & 0.01 \\
Deep venous thrombosis & $5(7)$ & $2(1)$ & 0.007 \\
Pulmonary emboli & $1(1)$ & $2(1)$ & 0.53 \\
Mortality hospital & $2(3)$ & $0(0)$ & 0.21 \\
Mortality 30 days & $7(10)$ & $2(1)$ & $<0.001$ \\
Mortality 365 days & $12(18)$ & $3(1)$ & $<0.001$ \\
Length of stay, median days (Q1-Q3) & $7.0(4.0-12.5)$ & $3.0(2.0-4.0)$ & $<0.001$ \\
Hospital costs, median US\$ (Q1-Q3) & $50844(31503-77730)$ & $14252(10003-23886)$ & $<0.001$ \\
\hline
\end{tabular}

ESRD, end-stage renal disease.

suggest that treatment recommendations included in societies' guidelines ${ }^{11}$ may not be applicable to patients with ESRD. First, we observed higher rates of volume overload suggesting that current recommendations for intravenous fluids resuscitation in DKA guidelines result in higher rates of fluid overload in the dialysis population. The main mechanism for the profound fluid and electrolyte depletion in DKA is osmotic diuresis, which is not present in dialysis patients with ESRD, increasing the risk of volume overload and pulmonary edema. ${ }^{13}$ Tzamaloukas $e a^{14}$ reported that the degree of hypertonicity and intracellular volume contraction is less profound in patients with ESRD compared with patients with normal renal function at similar hyperglycemia levels.

We suggest a modified approach for fluid replacement in patients with ESRD, preferring a small bolus of $250 \mathrm{~mL}$ initially with reassessment after each infusion-particularly if no hypotensive, or just insulin therapy without fluids bolus. ${ }^{13}$ Intensive fluid resuscitation during the initial management of DKA usually results in some degree of correction of hyperglycemia due to forced osmotic diuresis. However, hypertonicity in these patients is more a reflection of the degree of hyperglycemia and not due to osmotic diuresis, as previously discussed. ${ }^{13}$ It has been proposed that adequate insulin administration alone, with limited or without intravenous fluid resuscitation, may resolve the metabolic disturbances without complications. ${ }^{13}$ Current guidelines recommend an insulin infusion with a glucose correction rate of $50-75 \mathrm{mg} / \mathrm{dL} /$ hour. Using continuous glucose monitoring on patients with diabetes, Kazempour-Ardebili et $a l^{15}$ showed lower mean glucose levels by -36 to $-190 \mathrm{mg} / \mathrm{dL}$ during dialysis days compared with days off dialysis; and Sobngwi $e t$ $a l^{16}$ showed that total daily insulin needs can decrease by $\sim 25 \%$ on hemodialysis days. Hence, we suggest a careful and slower rate of hyperglycemia correction, considering the glucose-lowering effect of dialysis and/or potentially avoiding the use of intravenous insulin bolus on starting the insulin infusion, which may decrease the risk of hypoglycemia. However, future studies are needed to determine the best hydration regimen to manage patients with DKA and ESRD to minimize iatrogenic fluid overload.

Potassium concentration is maintained in the body by balancing the intake with the excretion and the transcellular shifts of potassium. Approximately $75 \%$ of patients admitted with DKA present with elevated serum potassium levels on admission owing to the shift of intracellular potassium to the extracellular compartment in the setting of hypertonicity, insulin deficiency and acidosis. ${ }^{517}$ As expected, patients with ESRD in this study presented with higher potassium levels due to diminished potassium excretion by the kidneys. ${ }^{17} 18$ During insulin treatment, potassium concentration rapidly decreases due to intracellular shifting of potassium, mediated by insulin stimulation of $\mathrm{Na}^{+}-\mathrm{H}^{+}$transporter, promoting the entry of $\mathrm{Na}^{+}$, then activation of the $\mathrm{Na}^{+}-\mathrm{K}^{+}$ATPase, resulting in influx of potassium ${ }^{19}$; as well as increased kaliuresis, thought to be mediated by an aldosterone-like effect of insulin. ${ }^{20}$ In recent studies in patients with ESRD, the prevalence of hyperkalemia $\geq 5 \mathrm{mmol} / \mathrm{L}$ was reported between $14 \%$ and $20 \%$, and the prevalence of hypokalemia $\leq 4 \mathrm{mmol} / \mathrm{L}$ was between $12 \%$ and $18 \%,{ }^{17}$ with both conditions associated with increased mortality. ${ }^{21}$ Patients with DKA and ESRD presented with higher potassium concentration compared with patients with preserved renal function. In agreement with previous reports, insulin administration was the only treatment required for correction of hyperkalemia. ${ }^{18}{ }^{21}$ Of interest, we observed no differences in the rate of hypokalemia between groups. These results indicate the need for a modified potassium administration approach in the management of DKA in patients with ESRD, as well as close follow-up of potassium levels on admission and during insulin treatment.

We acknowledge several limitations in our analysis including a relatively small number of patients with ESRD presenting with DKA; however, this is the largest 
cohort study of patients with ESRD admitted with DKAan uncommon scenario. ${ }^{10}$ The retrospective nature of the study and the use of ICD-9 codes may limit the ability to accurately differentiate between type 1 and type 2 diabetes, duration of diabetes and precipitant causes. Nonetheless, despite using ICD-9/10 codes, we were able to confirm all cases of DKA and ESRD based on biochemical data on admission, which have been a limitation of previous reports. In addition, although a DKA treatment protocol is available for use in the emergency department and in intensive care unit and step-down units, the current protocols are not designed to adjust for changes in insulin, fluid and electrolyte administration in patients with diabetes and ESRD on hemodialysis. We also recognized the limited value of HbA1c in the setting of ESRD.

In conclusion, we report significant metabolic and clinical outcome differences between patients with DKA and ESRD compared with patients with preserved renal function. Patients with DKA and ESRD on hemodialysis have higher rates of complications, increased resource utilization and hospitalization costs than patients with DKA and preserved renal function. We observed a greater number of patients with DKA and ESRD developing volume overload and hypoglycemia during insulin treatment compared with patients with preserved renal function. These results indicate that a personalized and careful approach to insulin and fluid replacement and correction of hyperglycemia is needed to improve outcome and prevent iatrogenic complications, volume overload and hypoglycemia.

Contributors RJG and GEU designed the study and wrote the manuscript. FJP, MF, KT, ND, SC, HW, and PV contributed to the study design, data collection, data analysis and reviewed/edited the manuscript. RJG and GEU are the guarantors of this work and, as such, had full access to all the data in the study and take responsibility for the integrity of the data and the accuracy of the data analysis.

Funding Research reported in this publication was supported by a grant to RJG from the National Institute of Diabetes and Digestive and Kidney Diseases of the National Institute of Health Under Award number P30DK11102. RJG received research support to Emory University for investigator-initiated studies from Novo Nordisk, and consulting fees from Abbott Diabetes Care, Sanofi and Novo Nordisk. PV is supported in part by the National Institute of Health grant 3K12HD085850 and has received consulting fees from Merck and Boehringer Ingelheim. FJP is supported in part by the National Institute of Health grant 1K23GM128221-01A1 and received consulting fees from Boehringer Ingelheim, Eli Lilly and AstraZeneca. GEU is partly supported by research grants from the National Center for Advancing Translational Sciences of the National Institutes of Health under Award number UL1TR002378 from the Clinical and Translational Science Award program and a National Institutes of Health (NIH) grant U30, P30DK11102, and has received research grant support to Emory University for investigator-initiated studies from Sanofi, Novo Nordisk, and Dexcom.

Patient consent for publication Not required.

Provenance and peer review Not commissioned; externally peer reviewed.

Data availability statement Data are available upon reasonable request. Study protocol and deidentified data that underlie the results of this article will be available upon reasonable request. Data will be available beginning 9 months and ending 12 months following article publication. We will share data with investigators whose proposed use of data has been approved by an independent review committee identified for this purpose.

Open access This is an open access article distributed in accordance with the Creative Commons Attribution 4.0 Unported (CC BY 4.0) license, which permits others to copy, redistribute, remix, transform and build upon this work for any purpose, provided the original work is properly cited, a link to the licence is given, and indication of whether changes were made. See: https://creativecommons.org/ licenses/by/4.0/.

\section{ORCID IDs}

Rodolfo J Galindo http://orcid.org/0000-0002-9295-3225

Priyathama Vellanki http://orcid.org/0000-0002-6544-015X

Guillermo E Umpierrez http://orcid.org/0000-0002-3252-5026

\section{REFERENCES}

1 Center for Disease Control and Prevention (CDC). Chronic kidney disease surveillance System-United states, 2017.

2 USRDS. Epidemiology of kidney disease in the United States. National Institutes of health, National Institute of diabetes and digestive and kidney diseases. 2017 USRDS annual data report, 2017.

3 Tuttle KR, Bakris GL, Bilous RW, et al. Diabetic kidney disease: a report from an ADA consensus conference. Diabetes Care 2014;37:2864-83.

4 Bell S, Fletcher EH, Brady I, et al. End-Stage renal disease and survival in people with diabetes: a national database linkage study. QJM 2015;108:127-34.

5 Umpierrez G, Korytkowski M. Diabetic emergencies - ketoacidosis, hyperglycaemic hyperosmolar state and hypoglycaemia. Nat Rev Endocrinol 2016;12:222-32.

6 Hurtado CR, Lemor A, Vallejo F, et al. Causes and predictors for 30day RE-ADMISSIONS in adult patients with diabetic ketoacidosis in the United States: a nationwide analysis, 2010-2014. Endocr Pract 2019;25:242-53.

7 Vellanki P, Umpierrez GE. Diabetic ketoacidosis: a common DEBUT of diabetes among African Americans with type 2 diabetes. Endocr Pract 2017;23:971-8.

8 Neumiller JJ, Alicic RZ, Tuttle KR. Therapeutic considerations for antihyperglycemic agents in diabetic kidney disease. J Am Soc Nephrol 2017;28:2263-74.

9 Desai D, Mehta D, Mathias P, et al. Health care utilization and burden of diabetic ketoacidosis in the U.S. over the past decade: a nationwide analysis. Diabetes Care 2018;41:1631-8.

10 Schaapveld-Davis CM, Negrete AL, Hudson JQ, et al. End-Stage renal disease increases rates of adverse glucose events when treating diabetic ketoacidosis or hyperosmolar hyperglycemic state. Clin Diabetes 2017;35:202-8.

11 Kitabchi AE, Umpierrez GE, Miles JM, et al. Hyperglycemic crises in adult patients with diabetes. Diabetes Care 2009;32:1335-43.

12 Galindo RJ, Davis GM, Fayfman M, et al. Comparison of efficacy and safety of Glargine and Detemir insulin in the management of inpatient hyperglycemia and diabetes. Endocr Pract 2017;23:1059-66.

13 Tzamaloukas AH, Ing TS, Siamopoulos KC, et al. Body fluid abnormalities in severe hyperglycemia in patients on chronic dialysis: review of published reports. J Diabetes Complications 2008;22:29-37.

14 Tzamaloukas AH, Ing TS, Siamopoulos KC, et al. Body fluid abnormalities in severe hyperglycemia in patients on chronic dialysis: theoretical analysis. J Diabetes Complications 2007;21:374-80.

15 Kazempour-Ardebili S, Lecamwasam VL, Dassanyake T, et al. Assessing glycemic control in maintenance hemodialysis patients with type 2 diabetes. Diabetes Care 2009;32:1137-42.

16 Sobngwi E, Enoru S, Ashuntantang G, et al. Day-To-Day variation of insulin requirements of patients with type 2 diabetes and end-stage renal disease undergoing maintenance hemodialysis. Diabetes Care 2010;33:1409-12.

17 Gilligan S, Raphael KL. Hyperkalemia and hypokalemia in CKD: prevalence, risk factors, and clinical outcomes. Adv Chronic Kidney Dis 2017;24:315-8.

18 Tzamaloukas AH, Ing TS, Elisaf MS, et al. Abnormalities of serum potassium concentration in dialysis-associated hyperglycemia and their correction with insulin: review of published reports. Int Urol Nephrol 2011;43:451-9.

19 DeFronzo RA, Felig P, Ferrannini E, et al. Effect of graded doses of insulin on splanchnic and peripheral potassium metabolism in man Am J Physiol Endocrinol Metab 1980;238:E421-7.

20 West ML, Magner PO, Richardson RMA, et al. A renal mechanism limiting the degree of potassium loss in severely hyperglycemic patients. Am J Nephrol 1988;8:373-8.

21 Nakhoul GN, Huang H, Arrigain S, et al. Serum potassium, endstage renal disease and mortality in chronic kidney disease. Am J Nephrol 2015;41:456-63. 